\title{
Correction to: Cannabimimetic activities of cumyl carboxamide-type synthetic cannabinoids
}

\author{
Akiko Asada ${ }^{1}$ (I) Takahiro Doi ${ }^{1} \cdot$ Takaomi Tagami $^{1} \cdot$ Akihiro Takeda $^{1} \cdot$ Yuka Satsuki $^{1} \cdot$ Masami Kawaguchi $^{1}$. \\ Akihiko Nakamura $^{1} \cdot$ Yoshiyuki Sawabe $^{1}$
}

Published online: 21 July 2020

(c) Japanese Association of Forensic Toxicology 2020

\section{Correction to: Forensic Toxicol (2018) 36:170-177 https://doi.org/10.1007/s11419-017-0374-9}

There were some mistakes in Table 1 (the values of $\mathrm{EC}_{50}$ of compounds 5, 6, and 7). The correct Table 1 is placed below.
Publisher's Note Springer Nature remains neutral with regard to jurisdictional claims in published maps and institutional affiliations.
Table 1 Half maximal (50\%) effective concentration $\left(\mathrm{EC}_{50}\right)$ values of cumyl carboxamidetype cannabinoid compounds

\begin{tabular}{llllll}
\hline Entry & Compound & \multicolumn{2}{l}{$\mathrm{EC}_{50}(\mathrm{~mol} / \mathrm{L})$} & & \\
\cline { 3 - 6 } & & $\mathrm{CB}_{1}$ & $\mathrm{CB}_{2}$ & $\mathrm{CB}_{1} / \mathrm{CB}_{2}$ & $\mathrm{CB}_{2} / \mathrm{CB}_{1}$ \\
\hline 1 & CUMYL-PINACA & $5.12 \times 10^{-9}$ & $4.75 \times 10^{-8}$ & 0.11 & 9.28 \\
2 & CUMYL-5F-PINACA & $1.51 \times 10^{-8}$ & $3.48 \times 10^{-8}$ & 0.43 & 2.30 \\
3 & CUMYL-PICA & $7.77 \times 10^{-7}$ & $4.44 \times 10^{-7}$ & 1.75 & 0.57 \\
4 & CUMYL-5F-PICA & $2.09 \times 10^{-8}$ & $9.09 \times 10^{-6}$ & $<0.01$ & 435 \\
5 & CUMYL-THPINACA & $8.14 \times 10^{-10}$ & $1.33 \times 10^{-9}$ & 0.61 & 1.63 \\
6 & CUMYL-BICA & $1.24 \times 10^{-9}$ & $3.95 \times 10^{-10}$ & 3.14 & 0.32 \\
7 & CUMYL-5F-P7AICA & $1.21 \times 10^{-9}$ & $2.48 \times 10^{-10}$ & 4.88 & 0.20 \\
8 & CP 55940 (positive control) & $7.35 \times 10^{-10}$ & $4.69 \times 10^{-10}$ & 1.57 & 0.64 \\
\hline
\end{tabular}

The original article can be found online at https://doi.org/10.1007/ s11419-017-0374-9.

Akiko Asada

asada@iph.osaka.jp

1 Osaka Institute of Public Health, 1-3-69 Nakamichi,

Higashinari-ku, Osaka 537-0025, Japan 